\title{
Research and Application of Machine Vision in Industry
}

\author{
Wei Liao ${ }^{1, a}$, Xiaorui Pei ${ }^{1, b}$
}

${ }^{1}$ Dept. of Mechanical Engineering, Wuhan University of Techlonogy, 430070, Wuhan, china

aLiaowei66666688@126.com, bx_p2009@126.com

Keywords: machine-vision, on-line inspection, geometry measurement

\begin{abstract}
This paper briefly introduce the concept and characteristic and form of machine vision inspecting system, elaborate its application in industry, the present status and development prospects of the application in geometry measurement are described in detail, and deal with emphatically its critical technical points and the corresponding solutions.
\end{abstract}

\section{Introduction}

Detecting is an absolutely necessary link in mechanical processing; modern manufacturing industry is developing toward automation, high speed and preciseness. People have higher and higher requirement on product processing. To determining whether the products are qualified and then taking appropriate actions, these products are simply detected and analyzed through measuring device and human visual in the traditional detecting. The traditional detecting method, with dependence of human, heavy labor intensity, low productivity, inefficiency, seriously restrains the development of advanced manufacturing. So there is a dire need for an automatic detection means to replace the manual inspection methods.

Some visual equipments, such as visual sensor(cmos and ccd), illumination system and computer, which simulate the human visual function, are mainly used in Machine vision, then obtain information, which is to be measured, from the images of target objects, and the intention to detect, measure and control is achieved.

Visual inspection is a non-contact detection, which is safe and reliable, will not result in any injury on the product and detector. In addition, machine vision has the advantages of high speed and high precision and automatic identification and detection. In comparison with the traditional detecting method, the machine vision has some strong points:

1. People will be freed from repeated and tedious work.

2. Reduced labor costs.

3. Put up productivity and detection accuracy.

4. To achieve flexible manufacturing.

Based on a lot of technical literature both abroad and domestic, this paper makes a brief description of machine vision systems and mainly discussed the detection of machine vision in industrial research and application.

\section{Introduction to visual inspection system}

The visual inspection system, which is in the application of visual sensor such as CMOS and CCD, can obtain the image of the measured target and then use Image processing techniques for non-contact detection. A typical structure of visual inspection system is shown in Figure 1. The main parts are as follows: illumination system, image acquisition devices, and image processing system, intelligent decision and execution system.

The design and arrangement of the illumination system is an important part of the visual inspecting system, and the reasonability can directly affect the success of the whole system. The illumination system should make the characters of the objects stand out as more as possible. There must be a striking contrast between the detecting parts and unimportant parts. In addition, the design and layout of the image acquire devices is also very important. At present, there are two kinds of 
image sensors: Charge Coupled Device (CCD) and CMOS sensor and these sensors are the main parts used for obtaining the original information of the object. After obtaining the distinct image of the object, then will be passed to image processing system for image processing.extract the character of the region-of-interest to describe and then provide a basis for deciding and then take corresponding actions.

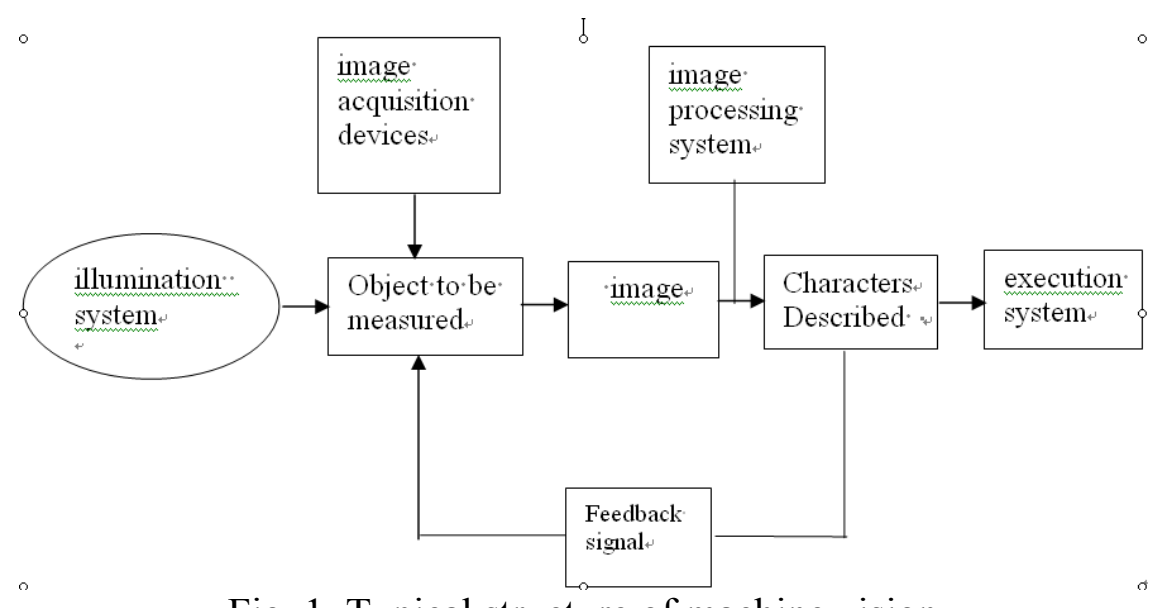

Fig. 1. Typical structure of machine vision

\section{The application of machine vision in industrial detection}

Though machine vision started later in industrial detection, it has been successfully applied to detection of industry product because of its outstanding qualities and increase productivity and quality of the products, also assure the stability of production.

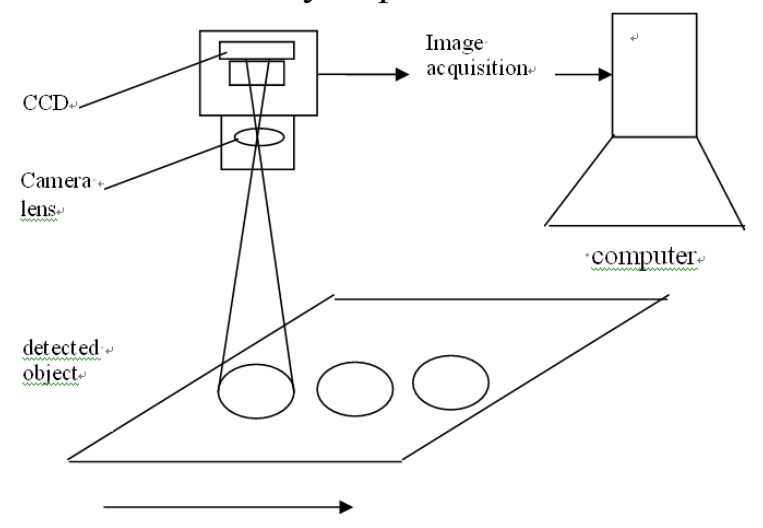

Fig. 2. The application of machine vision in industry

It has been mainly used for surface detection in electronic manufacturing, online quality inspecting in mechanical engineering, to improving the output and quality of products through feedback control. In the field of automobile, machine vision is used for assembly-line inspection and off-line detection of products and surface detection, it is used for packaging in the food industry, used for uniformity test and surface test in the field of paper products and Optimized the production processes at the same time. On pharmaceutical production line, machine vision is used for detecting medical package, then decide whether the number of loaded pills is correct. In a word ,the application of machine vision save a lot of manpower and material resources and lower the cost of product.

Nowadays, machine vision is most commonly used for the measurement of geometry of mechanical parts in industry inspection. such as ZheJiang University ${ }^{[1]}$, South China University of Technology ${ }^{[2]}$, the diameter of the circular components is detected by machine vision. Figure 2 shows the structure of the measurement system. Though they have their own characteristic in their measurement system, the approach and step they have taken are always the same, its mainly procedures include: system calibration, image acquisition, image preprocessing, image analysis and feature extraction ect. 
Though machine vision are widely used in geometrical measurement, according to many domestic and international literature shows that most detected objects are finished goods and remain relatively static( with respect to the support plate).Image acquisition and the establishment of inspecting system may become easier in these condition. However, once the finished product is not eligible, it is difficult to repair or re-processing, in this condition, it is not only a waste of time but also a waste of resources. With the improvement of automation, we need a manufacturing system with high degree of information, flexible and automation. Machine vision inspection is a non-contact detection, can be used for the detection of machining parts, that is to say, the machining parts is considered as a detected object. In this way, the geometry information can be feeded back to the worker or a manufacturing system, then adjust the amount of processing to assuring the quality of the product and achieve flexible manufacturing. But the online testing system compared with the offline testing system, still exists two difficult problems:

Get a distinct image of rotating parts with high speed

In the workshop, the processing environment is very complex, such as the vibration of the machine, the surface reflective of part, the brightness of the workshop and etc., all these conditions can affect the image collection. so it is necessary to design a reasonable system used for image collection, the system includes illumination system and imaging system. The system shall not only assure the time of lighting can match with the camera's exposure time, but also can automatically adjust according to the change of the machining condition.

Ensure the realtimeness of measurement

It makes severe demands on operating time and processing speed of the system, because the detected objects are rotating workpiece.

Mechanical hand are widely used in industry currently, which has the virtue of high precision and fast response. So according to first problem we can design a holding device similar to mechanical hand to support and control the camera. so the camera can get a instinct image when the camera move to a suitable place. The device include actuator and control systems. Control system is used to make camera get its most suitable place and obtaining a instinct image. Real-Time Problem include hard real-time problem and soft real-time problem. The first problem is solved by special industrial digital camera with high-performance and lighting, for the second, the velocity of image processing is mainly increased by improved algorithm

\section{Conclusion}

The application of machine vision in auto-measurement technique field is summed up in two methods-automatic detection of visual recognition and geometrical measurement testing. The research of the former come mature and is widely used. Most detected objects in the latter method is static, little research has been conducted in applying the latter method to dynamic object with high speed. So we explore new theories and methods on the basis of static test results at home and abroad and develop hardware and software systems of machine vision, then greatly improve the speed of image acquisition and processing, In this way, can meet the real-time need and improve the precision in the on-line inspection and realize flexible and automated production.

\section{References}

[1] Roland T.Chin, Charles,A. Harlow, Automated Visual Inspection: A Survey, J IEEE Transactions On Pattern Analysis And Machine Intelligence.6(1982)557-573.

[2] Fan Xiang, Lu Dao-hua, Wang Jia, Research and application of machine vision in industry, J.Modern Manufacturing Engineering,2007,6.

[3] Elias N. Malamas.A survey on industrial vision systems, applications and tools,J.Image and Vision Computing,21(2003)171-188.

[4] Lin Xiao-feng, Shi Shao-li. Size measurement of workpieces by using CCD measuring system, J. Optical Instrument,2002,24(3).

[5] He Ya-qin, Li Lin.Research for part size inspection base on machine vision, J. Coal Mine Machinery,2010,31(6). 